\title{
Revisão de Literatura
}

\section{Associação entre síndrome metabólica e transtornos mentais}

\section{Epidemiologic studies about association of mental disorders and metabolic syndrome}

\author{
Paulo José Ribeiro Teixeira ${ }^{1}$, Fábio Lopes Rocha ${ }^{2}$ \\ ' Psiquiatra e coordenador do Programa de Psiquiatria da Residência Médica do Instituto de Previdência dos Servidores do Estado de Minas Gerais (IPSEMG) e mestre em Ciências da Saúde pelo IPSEMG \\ 2 Psiquiatra e coordenador da Clínica Psiquiátrica e do Curso de Pós-graduação em Ciências da Saúde do IPSEMG e doutor em Ciências da Saúde pela Universidade de Brasilia (UnB).
}

Recebido: 16/02/2006 - Aceito: 28/06/2006

\section{Resumo}

Contexto: A síndrome metabólica (SM) é um transtorno de alta prevalência na população geral. Estudos que demonstram haver associação positiva entre doenças mentais e diabetes melito e a constatação dos efeitos adversos metabólicos dos psicofármacos levantam a hipótese de que a prevalência seja ainda maior em pacientes psiquiátricos. Objetivo: O objetivo desta revisão é avaliar os estudos epidemiológicos sobre a associação entre os transtornos mentais e a SM ou seus componentes. Métodos: Foi realizada busca nas bases Medline e Lilacs, com a inclusão no estudo de artigos que apresentaram dados epidemiológicos sobre SM ou seus componentes em populações psiquiátricas. Resultados: Indivíduos com esquizofrenia ou transtornos esquizoafetivos apresentaram prevalência de SM variando entre 28,4\% e 62,5\%; um estudo com indivíduos esquizofrênicos mais jovens apresentou prevalência menor, de 19,4\%, porém 3,7 vezes maior que a do grupo-controle. Um estudo avaliou pacientes com transtorno afetivo bipolar e verificou prevalência de $30 \%$. Prevalência elevada foi encontrada em mulheres com história de depressão, mas não em homens. Conclusão: A prevalência de SM é elevada em pacientes com esquizofrenia e transtornos esquizoafetivos e em mulheres com história de depressão.

Teixeira, P.J.R.; Rocha, F.L. / Rev. Psiq. Clín. 34 (1); 28-38, 2007

Palavras-chave: Síndrome metabólica, transtornos mentais, esquizofrenia, transtorno bipolar, depressão.

\section{Abstract}

Background: Metabolic syndrome (MS) is a very prevalent condition. Studies that show a positive association between mental diseases and diabetes mellitus and the existence of adverse metabolic effects caused by psychotropic drugs allow the presumption that the occurrence of MS is even greater in psychiatric patients. Objective: The objective of this review is to assess the epidemiological studies about the relationship between MS or its components and mental disorders. Methods: Research was carried out at Medline and Lilacs, searching for articles which presented epidemiological data on the prevalence or incidence of MS and its components in psychiatric populations. Results: Schizophrenic patients or with schizoaffective disorder demonstrated an increased prevalence of MS, between $28,4 \%$ and $62,5 \%$; one study, with younger schizophrenic patients, showed a prevalence of 19,4\%, however 3.7 times higher than the controls. One study with patients with bipolar disorder showed a prevalence of $30 \%$. Increased prevalence of MS was found in women with a history of depression, but not in men. Conclusion: The prevalence of MS is high in patients with schizophrenia and schizoaffective disorder, and in women with a history of depression.

Teixeira, P.J.R.; Rocha, F.L. / Rev. Psiq. Clín. 34 (1); 28-38, 2007

Key-words: Metabolic syndrome, mental disorders, schizophrenia, bipolar disorder, depression. 


\section{Introdução}

A associação de uma dieta excessivamente calórica e rica em lipídeos com pouca atividade física, tão comum em nossos dias, tem resultado em uma epidemia mundial de obesidade e de doenças ligadas ao metabolismo da glicose (Zimmet et al., 2001). Entre as doenças ligadas ao metabolismo da glicose, destaca-se a síndrome metabólica (SM), um transtorno complexo formado por um conjunto de fatores de risco cardiovascular usualmente relacionado à deposição central de gordura e à resistência à insulina (Reaven, 1994; Rosenbaum e Ferreira, 2003). Seus principais componentes são: a obesidade central, as alterações da homeostase da glicose, como hiperglicemia ou diabetes melito (DM) tipo II, a elevação da pressão arterial e a dislipidemia. Estados pró-inflamatório e pró-trombótico também estão presentes (Grundy et al., 2004).

Definições foram propostas para a SM (Tabela 1). A primeira delas, da Organização Mundial da Saúde (OMS), considera a resistência insulínica como fator indispensável a seu diagnóstico (Alberti e Zimmet, 1998). Uma segunda definição foi elaborada posteriormente pelo National Cholesterol Education Program - Adult Treatment Panel III (NCEP-ATP III) e, por apresentar maior aplicabilidade clínica, utilizada na maioria dos estudos (Summary of the third report of the National Cholesterol Education Program - NCEP-ATP III, 2001). Mais recentemente, a
Federação Internacional de Diabetes (IDF) propôs nova definição, na qual a presença de gordura visceral torna-se o principal fator diagnóstico (IDF, 2005).

ASM é um transtorno de alta prevalência na população em geral. Nos Estados Unidos, a prevalência ajustada para a idade na população adulta é de $23,7 \%$, segundo dados do Third National Health and Nutrition Examination Survey - NHANES III (Ford et al., 2002). Não há estudos de prevalência representativos da população brasileira (Sociedade Brasileira de Hipertensão et al., 2004).

A associação entre transtornos psiquiátricos e distúrbios metabólicos é aventada de longa data. Ainda no final do século XIX, sir Henry Maudsley, eminente psiquiatra inglês, afirmou que "o diabetes é uma doença freqüentemente presente em famílias nas quais também prevalece a insanidade" (Holt et al., 2004).

Revisão de prontuários de pacientes com diagnóstico de esquizofrenia ou transtorno afetivo bipolar (TAB), admitidos em hospital psiquiátrico nos anos de 1940 a 1950, constatou taxas elevadas de diabetes, hipertensão e excesso de peso (Bellnier, 2003). Ademais, indivíduos com transtornos mentais constituem um grupo de morbimortalidade elevada. A taxa de mortalidade padronizada de indivíduos com esquizofrenia é de 1,5 a 4 vezes aquela da população em geral, e óbitos por causas naturais respondem por quase dois terços desse excesso, com elevação significativa dos

Tabela 1. Definições para a síndrome metabólica

OMS

NCEP-ATP III

1. Três ou mais das seguintes condições:

a. obesidade central (circunferência abdominal $>102 \mathrm{~cm}$ para homens e $>88 \mathrm{~cm}$ para mulheres)

b. trigliceridemia $\geq 150 \mathrm{mg} / \mathrm{dl}$

c. níveis de $\mathrm{HDL}<40 \mathrm{mg} / \mathrm{dl}$ para homens e $<50 \mathrm{mg} / \mathrm{dl}$ para mulheres

d. pressão arterial $\geq 130 / \geq 85 \mathrm{mmHg}$

e. glicemia de jejum $\geq 110 \mathrm{mg} / \mathrm{dl}$

1. Alteração da regulação da glicose (tolerância alterada à glicose ou à glicemia de jejum alterada) ou DM e/ou resistência à insulina

Duas ou mais das seguintes condições:

$(\mathrm{IMC})>30 \mathrm{~kg} / \mathrm{m}^{2}$

d. microalbuminúria (taxa de excreção urinária de albumina $\geq 20 \mathrm{mcg} / \mathrm{min}$ ou índice albumina/creatinina $\geq 20 \mathrm{mg} / \mathrm{g}$ )

IDF

1. Obesidade central (circunferência abdominal $\geq 94 \mathrm{~cm}$ para homens europeus ou $\geq 88 \mathrm{~cm}$ para mulheres européias)

Valores específicos devem ser usados para outros grupos étnicos

2. Duas ou mais das seguintes condições:

a. trigliceridemia $\geq 150 \mathrm{mg} / \mathrm{dl}$ ou tratamento específico para essa anormalidade lipídica

b. níveis sangüíneos de $\mathrm{HDL}<40 \mathrm{mg} / \mathrm{dl}$ para homens ou $<50 \mathrm{mg} / \mathrm{dl}$ para mulheres, ou tratamento específico para essa anormalidade lipídica

c. pressão sistólica $\geq 130 \mathrm{mmHg}$ ou pressão diastólica $\geq 85 \mathrm{mmHg}$, ou tratamento específico para hipertensão previamente diagnosticada

d. glicemia de jejum $\geq 100 \mathrm{mg} / \mathrm{dl}$, ou diagnóstico prévio de DM tipo II 
indicadores de mortalidade relacionados ao DM e às doenças cerebrovasculares (Brown, 1997; Brown et al., 2000; Harris e Barraclough, 1988; Jeste et al., 1996).

Pacientes com transtornos do humor, por sua vez, apresentam taxa de mortalidade padronizada 50\% maior que a da população em geral, e causas naturais respondem por 45\% desse excesso (Harris e Barraclough, 1988; Murphy et al., 1987). A depressão, per se, confere um risco relativo de 1,5 a 2,5 vezes para morbidade e mortalidade por causas cardíacas (Lett et al., 2004). Por fim, muitos psicofármacos apresentam efeitos colaterais importantes relacionados à SM, como ganho de peso, alterações no metabolismo da glicose e dislipidemias (ADA et al., 2004; Casey et al., 2004a ou b; Fava, 2000; Garland et al., 1988). Face a esses dados, é razoável supor que a prevalência de SM seja maior na população psiquiátrica, quando comparada à prevalência na população geral.

Assim, o objetivo do presente estudo é realizar uma revisão da literatura médica buscando estudos epidemiológicos que avaliaram a prevalência ou a incidência de SM em pacientes portadores de transtornos mentais, bem como dos distúrbios metabólicos que a constituem.

\section{Métodos}

Foi realizada pesquisa nas bases eletrônicas de dados Medline e Lilacs, buscando artigos que tratassem da associação entre SM e transtornos mentais. Na Medline, utilizou-se a expressão "metabolic syndrome”, associada às seguintes, em todos os campos: "mental illness", "mental disorder", "psychiatric illness", "psychiatric disorder", "schizophrenia”, "bipolar disorder", "depression” e "mood disorder". Na base de dados Lilacs, foram utilizados os descritores "síndrome x metabólica”, "diabetes" e "hiperglicemia”, associados aos seguintes: "transtornos mentais", "esquizofrenia”, "transtorno bipolar" e "depressão". Estudos relevantes não encontrados na busca inicial foram selecionados a partir da lista de referências dos artigos encontrados. Foram incluídos no estudo todos os artigos em línguas portuguesa, inglesa ou espanhola que apresentassem dados epidemiológicos sobre prevalência ou incidência de SM e seus componentes em indivíduos com transtornos mentais. Foram excluídos desta revisão sistemática os artigos que tinham como objetivo principal investigar a associação de psicofármacos com a SM ou com os distúrbios que a compõem.

\section{Resultados}

Dezesseis estudos epidemiológicos sobre prevalência de SM em populações psiquiátricas foram encontrados nesta revisão da literatura. Outros 24 estudos apresentaram dados de prevalência ou incidência de distúrbios constituintes da SM (obesidade, distúrbios do metabolismo da glicose, hipertensão arterial e dislipidemias) em populações psiquiátricas.

\section{Transtornos mentais e síndrome metabólica}

Três estudos avaliaram populações psiquiátricas sem diagnóstico específico e verificaram taxas de prevalência de 29,2\% (Straker et al., 2005), 37,3\% (Correll et al., 2006) e 46\% (Crabtree, 2004). Pacientes com esquizofrenia ou transtorno esquizoafetivo foram objetos de onze estudos, e, em nove deles, a prevalência de $\mathrm{SM}$ pelos critérios do NCEP-ATP III variou entre 28,4\% e 62,5\% (Basu et al., 2004; Cohn et al., 2004; De Hert et al., 2006; Hagg et al., 2006; Heiskanen et al., 2003; Kato et al., 2004; McEvoy et al., 2005; Meyer et al., 2006; Pandina et al., 2004). Destes, o estudo realizado na Holanda apresentou a menor prevalência de SM (28,4\%); todavia, esse valor foi mais de duas vezes a prevalência de SM na população (De Hert et al., 2006). Os dois estudos restantes encontraram taxas inferiores a esses valores (Littrell et al., 2003; Saari et al., 2005). Um deles utilizou critérios diagnósticos próprios, não descritos pelos autores, e constatou prevalência de $22,2 \%$ em pacientes internados (Littrell et al., 2003). O outro estudo avaliou indivíduos com esquizofrenia participantes do Northern Finland 1966 Birth Cohort e obteve prevalência de 19,4\%, inferior aos valores citados, mas 3,7 vezes maior que a do grupo-controle (Saari et al., 2005). Nesse estudo, a prevalência de SM em indivíduos com outros tipos de psicose ou com transtornos mentais não psicóticos não foi mais elevada que a prevalência nos controles.

Um dos estudos que avaliaram a prevalência de SM em indivíduos com esquizofrenia utilizou dados de 689 pacientes incluídos no Clinical Antipsychotic Trial of Intervention Effectiveness (CATIE). O CATIE é um ensaio clínico patrocinado pelo National Institute of Mental Health (NIMH) que tem por objetivo comparar a efetividade de quatro antipsicóticos atípicos e um antipsicótico tradicional. A prevalência encontrada, pela definição do NCEP, foi de 40,9\%, sendo significativamente maior em mulheres do que em homens (respectivamente, 51,6\% e 36,0\%, $\mathrm{p}=0,002)$. Quando comparados a controles do Third National Health and Nutrition Examination Survey (NHANES III), a prevalência de SM foi significativamente maior tanto para homens (OR: 2,297, $\mathrm{p}<0,0001$ ) quanto para mulheres (OR: 3,186, $\mathrm{p}<0,0001)$. 
Um único estudo foi encontrado envolvendo pacientes com TAB, com prevalência de 30\% para SM (Fagiolini et al., 2005). Dos 171 pacientes examinados, $44 \%$ estavam em uso de lítio e 34\%, em uso de antipsicóticos atípicos. Não foram verificados estudos envolvendo pacientes com diagnóstico atual de depressão unipolar. Todavia, um estudo avaliou a prevalência de SM em indivíduos participantes do NHANES III, com história de episódio depressivo maior (Kinder et al., 2004). Em mulheres jovens (17 a 39 anos), a prevalência encontrada foi de $12,3 \%$, taxa duas vezes maior que a do grupo-controle. Esse aumento permaneceu significativo mesmo se considerados fatores de confusão, como sexo, etnia, grau de instrução, tabagismo, atividade física, consumo de carboidratos e de álcool. No entanto, nesse mesmo estudo, a prevalência de 11,7\% encontrada em homens da mesma faixa etária não diferiu significativamente daquela do grupo-controle.

Os estudos sobre prevalência de SM em pacientes com transtornos mentais encontram-se descritos na tabela 2. Foram publicados a partir de 2003 e envolveram

Tabela 2. Estudos de prevalência de síndrome metabólica em populações com transtornos mentais

\begin{tabular}{|c|c|c|c|c|c|}
\hline Estudo & $\begin{array}{c}\mathbf{N} \text { total } \\
\text { (mulheres) }\end{array}$ & Idade média (dp) & Diag. & Prevalência & Observações \\
\hline Heiskanen et al., 2003 & $35(16)$ & $45(10,4)$ & ESO & $37,1 \%$ & $\begin{array}{l}\text { Pacientes em uso de antipsicóticos } \\
\text { (50\% em uso de clozapina) }\end{array}$ \\
\hline Littrell et al., 2003 & $125(50)$ & Não informada & $\begin{array}{l}\text { ESQ/ } \\
\text { TEA }\end{array}$ & $\begin{array}{l}\text { Internados: } 22,2 \% \\
\text { Ambulatoriais: } 51,0 \%\end{array}$ & $\begin{array}{l}\text { Critérios para diagnóstico de SM } \\
\text { não foram informados pelos autores }\end{array}$ \\
\hline Basu et al., 2004 & $35(19)$ & $44,5(7,8)$ & TEA & $42,4 \%$ & $\begin{array}{l}\text { Dados da avaliação inicial de estudo } \\
\text { com topiramato }\end{array}$ \\
\hline Cohn et al., 2004 & $240(84)$ & $\begin{array}{l}\text { Homens: } 42,7(11,5) \\
\text { Mulheres: } 44,5(10,9)\end{array}$ & $\begin{array}{l}\text { ESQ/ } \\
\text { TEA }\end{array}$ & $\begin{array}{l}\text { Homens: } 42,6 \% \\
\text { Mulheres: } 48,5 \%\end{array}$ & - \\
\hline Crabtree, 2004 & 105 & Não informada & SDE & $46 \%$ & - \\
\hline Kato et al., 2004 & $48(24)$ & $40,3(12,0)$ & ESO & $62,5 \%$ & - \\
\hline Kinder et al., 2004 & $545(368)$ & $\begin{array}{l}\text { Homens: } 28,2 \\
\text { Mulheres: } 29,8\end{array}$ & $\begin{array}{l}\text { História } \\
\text { de EDM }\end{array}$ & $\begin{array}{c}\text { Homens: } 11,7 \% \\
\text { (OR: } 1,6 \text { 95\% IC: } 0,6-4,0) \\
\text { Mulheres: } 12,3 \% \\
\text { (OR: } 2,1 \text { 95\% IC: } 1,2-3,7 \text { ) }\end{array}$ & $\begin{array}{l}\text { Estudo controlado, com } \\
\text { dados do NHANES III }\end{array}$ \\
\hline Pandina et al., 2004 & 121 & Não informada & $\begin{array}{l}\text { ESQ/ } \\
\text { TEA }\end{array}$ & $54 \%$ & $\begin{array}{l}\text { Dados de avaliação de pacientes } \\
\text { refratários ou intolerantes à olanzapina }\end{array}$ \\
\hline Saari et al., 2005 & 31 & 31 anos & ESO & $\begin{array}{c}19,4 \% \\
\text { (OR: } 3,795 \% \text { IC: } 1,5-9,0)\end{array}$ & $\begin{array}{l}\text { Estudo controlado, com pacientes e } \\
\text { controles nascidos em } 1966\end{array}$ \\
\hline Straker et al., 2005 & $89(44)$ & $39,8(15,3)$ & SDE & $29,2 \%$ & $\begin{array}{l}\text { Pacientes em uso de } \\
\text { antipsicóticos atípicos }\end{array}$ \\
\hline McEvoy et al., 2005 & $689(180)$ & $40,4(11,2)$ & ESO & $\begin{array}{c}40,9 \% \text { (homens: } 36,0 \%- \\
\text { mulheres: } 51,6 \%, p=0,003 \text { ) }\end{array}$ & $\begin{array}{c}\text { Avaliação inicial de pacientes do } \\
\text { estudo CATIE }\end{array}$ \\
\hline Fagiolini et al., 2005 & $171(104)$ & $46,9(16,5)$ & $\mathrm{TAB}$ & $30 \%$ & - \\
\hline Meyer et al., 2006 & $80(6)$ & 49,0 & $\begin{array}{l}\text { ESQ/ } \\
\text { TEA }\end{array}$ & $51,2 \%$ & Prevalência ajustada para a idade \\
\hline Correll et al., 2006 & $367(169)$ & $42,9(15,3)$ & SDE & $37,3 \%$ & $\begin{array}{l}\text { Pacientes em uso de } \\
\text { antipsicóticos atípicos }\end{array}$ \\
\hline De Hert et al., 2006 & $430(151)$ & $36,5(11,8)$ & ESO & $\begin{array}{c}28,4 \% \text { (36\% pelos critérios } \\
\text { da IDF) }\end{array}$ & $\begin{array}{l}\text { Prevalência duas vezes maior do } \\
\text { que a da população de referência }\end{array}$ \\
\hline Hagg et al., 2006 & 269 & & ESO & $34,6 \%$ & - \\
\hline
\end{tabular}

N: tamanho da amostra; dp: desvio-padrão; Diag.: diagnóstico; SDE: sem diagnóstico específico; ESQ: esquizofrenia; TEA: transtorno esquizoafetivo; EDM: episódio depressivo maior; TAB: transtorno afetivo bipolar; OR: odds ratio; IC: intervalo de confiança; NHANES III: Third National Health and Nutrition Examination Survey; CATIE: Clinical Antipsychotic Trial of Intervention Effectiveness. 
populações da Bélgica (De Hert et al., 2006), do Canadá (Cohn et al., 2004), dos Estados Unidos (Basu et al., 2004; Crabtree, 2004; Kato et al., 2004; Kinder et al., 2004; Pandina et al., 2004; Fagiolini et al., 2005; McEvoy et al., 2005; Straker et al., 2005; Correll et al., 2006; Meyer et al., 2006), da Finlândia (Heiskanen et al., 2003; Saari et al., 2005) e da Suécia (Hagg et al., 2006). Um estudo avaliou conjuntamente pacientes dos Estados Unidos e de Taiwan (Littrell et al., 2003). No Brasil, ainda não foram realizados estudos dessa natureza.

\section{Transtornos mentais e obesidade}

Foram encontrados dez estudos com dados sobre prevalência de excesso de peso e obesidade em pacientes com transtornos mentais, sendo um deles uma revisão sistemática que abordou especificamente obesidade e transtornos de humor.

Susce et al. (2005) avaliaram 560 pacientes do estado norte-americano de Kentucky, portadores de transtornos psiquiátricos graves, e constataram prevalência de obesidade muito superior à da população local (OR: 2,6 - 95\% IC: 2,2-3,0). Sexo feminino, etnia afro-americana, uso prolongado ou início precoce de uso de psicofármacos associaram-se significativamente à obesidade.

Estudos em pacientes com esquizofrenia e transtorno esquizoafetivo apresentaram resultados divergentes. Prevalência aumentada de obesidade não foi encontrada em indivíduos com esquizofrenia residentes em Southampton, na Inglaterra (Brown et al., 1999). Análise dos dados do National Health Interview Survey, nos Estados Unidos, constatou que, comparados a controles, somente indivíduos esquizofrênicos do sexo feminino apresentavam elevação significativa do índice de massa corporal (IMC) (Allison et al., 1999a). Já a análise de dados basais de 420 pacientes com esquizofrenia ou transtorno esquizoafetivo, participantes de um ensaio clínico com ziprasidona, não evidenciou elevação significativa do IMC, quando comparados aos valores de referência da população americana (Allison et al., 1999a). Todavia, Theisen et al. (2001) encontraram taxas elevadas de obesidade em pacientes jovens com diagnóstico de esquizofrenia internados em um centro de reabilitação na Alemanha. Por fim, pacientes esquizofrênicos incluídos no estudo CATIE apresentaram valores médios elevados de IMC $\left(28,8 \mathrm{~kg} / \mathrm{m}^{2}\right.$ para homens e $32,8 \mathrm{~kg} / \mathrm{m}^{2}$ para mulheres); os autores, contudo, não informaram se esses valores diferiram significativamente das médias de IMC de indivíduos da mesma faixa etária na população (McEvoy et al., 2005).
Prevalência aumentada de obesidade ocorre em pacientes com transtorno de humor, principalmente naqueles com TAB. Um estudo controlado com 89 pacientes eutímicos portadores de $\mathrm{TAB}$ verificou prevalência de excesso de peso e obesidade significativamente maiores em mulheres (respectivamente, $44 \%$ versus $25 \%$ e $20 \%$ versus $13 \%$ ). Homens com TAB apresentaram prevalência significativamente maior de obesidade, mas não de excesso de peso (respectivamente, $18 \%$ versus $10 \%$ e $28 \%$ versus $43 \%$ ). Obesidade central foi significativamente maior em ambos os sexos (Elmslie et al., 2000). Dois outros estudos também apresentaram dados de prevalência de obesidade em pacientes com TAB, sendo encontradas taxas de $45 \%$ (Fagiolini et al., 2000) e 32\% (Fagiolini et al., 2005).

Um estudo prospectivo com homens de meia-idade (45 a 59 anos) não verificou associação entre IMC e sintomas depressivos (Lawlor et al., 2005). Por fim, revisão sistemática sobre a associação entre obesidade e transtornos do humor concluiu, pela análise dos estudos clínicos, que crianças e adolescentes com transtorno depressivo maior apresentam risco aumentado para o desenvolvimento de excesso de peso e que pacientes com $\mathrm{TAB}$ apresentam índices elevados de excesso de peso, obesidade e obesidade central (McElroy et al., 2004). Nessa mesma revisão, a análise de estudos baseados em comunidade constatou que depressão maior em mulheres associa-se positivamente à obesidade e que depressão atípica em mulheres associa-se a risco significativamente maior de excesso de peso, se comparada à depressão sem sintomas atípicos (McElroy et al., 2004).

Transtornos mentais e distúrbios do metabolismo da glicose

Cinco estudos avaliaram a prevalência de DM em indivíduos com esquizofrenia; dois deles também avaliaram pacientes com transtorno esquizoafetivo (Cohen et al., 2006; Dixon et al., 2000; Mukherjee et al., 1996; Regenold et al., 2002; Subramaniam et al., 2003).

Mukherjee et al. (1996) constataram prevalência de $15,8 \%$ para DM em pacientes com esquizofrenia de 45 a 74 anos internados em instituição de longa permanência na Itália. Dixon et al. (2000) analisaram dados do Schizophrenia Patient Outcomes Research Team (PORT) e encontraram prevalência de diabetes de $10,8 \%$, bem acima da taxa detectada no NHANES III $(1,2 \%$ para a faixa etária de 18 a 44 anos e 6,3\% para a de 45 a 64 anos). Regenold et al. (2002) constataram prevalência de 50\% para DM tipo II em pacientes internados com transtorno esquizoafetivo, taxa significativamente maior que 
aquela detectada no NHANES III. Encontraram também prevalência de $13 \%$ em pacientes com esquizofrenia, que, embora elevada, não diferiu significativamente do valor de referência. Em Singapura, taxas elevadas de DM (16\%) e tolerância alterada à glicose $(30,9 \%)$ foram verificadas em amostra de 194 pacientes com esquizofrenia residentes em instituição de longa permanência (Subramaniam et al., 2003). Na Holanda, Cohen et al. (2006) avaliaram 200 pacientes com diagnóstico de esquizofrenia ou transtorno esquizoafetivo e encontraram prevalência de $14,5 \%$ para DM, significativamente mais elevada que a prevalência ajustada para a idade na população holandesa $(1,5 \%)$.

Tomados em conjunto, esses estudos são concordantes em apontar índices elevados de DM em indivíduos com esquizofrenia.

Dois estudos controlados avaliaram alterações da homeostase da glicose em pacientes esquizofrênicos virgens de tratamento (Arranz et al., 2004; Ryan et al., 2003). Ryan et al. (2003) encontraram prevalência de $15 \%$ para glicemia de jejum alterada, comparada a $0 \%$ no grupo-controle; os pacientes com esquizofrenia também apresentaram insulinemia significativamente mais elevada e maior resistência à insulina, medida pelo índice HOMA. Todavia, Arranz et al. (2004) encontraram resultados divergentes. Esses autores avaliaram 100 pacientes divididos em dois grupos, com ou sem história de uso prévio de antipsicóticos, e os compararam a um terceiro grupo de 50 indivíduos saudáveis. Somente os pacientes com passado de uso de antipsicóticos apresentaram aumento significativo dos níveis séricos de insulina e peptídeo $\mathrm{C}$ e grau significativamente mais alto de resistência à insulina, medido pelo índice HOMA.

A prevalência de DM é elevada também em indivíduos com TAB. Cassidy et al. (1999) encontraram prevalência de $9,9 \%$ em amostra de 345 pacientes hospitalizados, significativamente superior ao valor de $3,4 \%$ da população de referência; os pacientes diabéticos apresentaram número maior de internações que os nãodiabéticos, embora o tempo de duração da doença fosse similar. Regenold et al. (2002) constataram prevalência de $26 \%$ para DM tipo II entre pacientes internados com $\mathrm{TAB}$, valor significativamente maior do que o detectado pelo NHANES III.

Estudos em amostras de pacientes deprimidos apresentaram resultados divergentes. Análise dos dados do Epidemiologic Catchment Area Project (ECA) não evidenciou aumento significativo do risco de desenvolvimento de DM em pacientes deprimidos (Eaton et al.,
1996). Em sentido contrário, outro estudo prospectivo, com seguimento médio de quinze anos, concluiu que a presença de grande número de sintomas depressivos é fator de risco para o surgimento de diabetes (RR: 1,86 - 95\% IC: 1,27-2,71), mesmo se considerados outros fatores, como uso de álcool, tabaco, atividade física e IMC (Carnethon et al., 2003). Regenold et al. (2002) verificaram prevalência de $18 \%$ para DM tipo II em pacientes internados com diagnóstico de depressão maior, valor que não diferiu significativamente se comparado ao da população de referência.

Estudos sobre a associação positiva entre resistência à insulina e depressão também apresentaram resultados contraditórios. Okamura et al. (2000) avaliaram, prospectivamente, vinte pacientes não-diabéticos e concluíram que indivíduos deprimidos apresentam menor sensibilidade à insulina, que tende a se normalizar com o tratamento da depressão. Everson-Rose et al. (2004) analisaram dados de 2.662 mulheres não-diabéticas e encontraram associação positiva entre depressão e resistência à insulina $(\mathrm{p}<0,04)$ e aumento na incidência de DM tipo II $(\mathrm{p}<0,03)$ em pacientes deprimidas para um período de três anos. Timonen et al. (2005) encontraram, em 491 pacientes com tolerância alterada à glicose, associação positiva entre gravidade dos sintomas depressivos, avaliada pelo Inventário de Beck, e maior resistência à insulina. Dois estudos contradisseram esses achados. Lawlor et al. (2003) mensuraram a resistência à insulina em mais de quatro mil mulheres com idade superior a 60 anos e encontraram associação em "J" entre resistência à insulina e depressão, com a prevalência desta última diminuindo com o aumento da resistência à insulina e passando a se elevar com o diagnóstico de diabetes. Mais recentemente, o mesmo grupo de pesquisadores não verificou correlação entre resistência à insulina e escores elevados de sintomas depressivos em estudo com 2.203 homens de meia-idade (Lawlor et al., 2005).

Como seria de se esperar, a prevalência de DM eleva-se em amostras de pacientes com transtornos psiquiátricos graves, sem diagnóstico específico. Susce $e t$ al. (2005) avaliaram pacientes dessa natureza do estado de Kentucky, sem diagnóstico específico, e encontraram prevalência de DM superior à esperada para a população local (OR: 2,9 - 95\% IC: 2,3-3,6).

\section{Transtornos mentais e elevação da pressão arterial}

É questionável a existência de associação entre transtornos mentais graves e elevação da pressão arterial, 
particularmente em se tratando de pacientes com esquizofrenia. Susce et al. (2005) não evidenciaram associação entre doença mental grave e hipertensão arterial. Cohn et al. (2004) não encontraram índices elevados de hipertensão em pacientes esquizofrênicos. Os pacientes esquizofrênicos participantes do CATIE também não apresentaram níveis médios elevados de pressão arterial (McEvoy et al., 2005). Todavia, essa associação pode existir em pacientes com $\mathrm{TAB}$, visto que, em um estudo envolvendo 171 pacientes, 39\% deles apresentaram índices pressóricos $\geq 130 / 85 \mathrm{mmHg}$ (Fagiolini et al., 2005).

A presença de sintomas depressivos pode ser fator predisponente para o surgimento de hipertensão arterial. Dois estudos prospectivos avaliaram especificamente esse tema e constataram risco aumentado de hipertensão em pacientes com escores elevados de sintomas depressivos. O primeiro, com acompanhamento de 7 a 16 anos, concluiu que altos índices de ansiedade ou depressão associam-se à maior incidência de hipertensão em indivíduos brancos na faixa etária de 45 a 64 anos ou em indivíduos negros na faixa etária de 25 a 64 anos (Jonas $e t$ al., 1997). O segundo, com acompanhamento de homens jovens por cinco anos, também demonstrou que escores elevados de sintomas depressivos associam-se à maior incidência de hipertensão (Davidson et al., 2000). Lawlor $e t$ $a l$. (2005), entretanto, não verificaram associação positiva entre sintomas depressivos e níveis de pressão sistólica, seja em análise prospectiva, seja transversal.

\section{Transtornos mentais e dislipidemias}

Não foram encontrados estudos que abordassem especificamente a associação entre transtornos mentais e dislipidemias. Ademais, dados nesse sentido que puderam ser extraídos de alguns dos estudos citados anteriormente não permitem que se conclua se há ou não essa associação (Cohn et al., 2004; Fagiolini et al., 2005; Kinder et al., 2004; Lawlor et al., 2005; McEvoy et al., 2005).

Kinder et al. (2004) constataram que a porcentagem de indivíduos com história de depressão com níveis elevados de triglicerídeos ( $>150 \mathrm{mg} / \mathrm{dl}$ ) ou níveis baixos de HDL ( $<40 \mathrm{mg} / \mathrm{dl}$ para homens ou $50 \mathrm{mg} / \mathrm{dl}$ para mulheres) não era diferente dos controles. Lawlor et al. (2005) não encontraram associação entre escores elevados de depressão e os níveis de HDL ou triglicerídeos, seja em análise prospectiva, seja transversal. A média da trigliceridemia de pacientes incluídos no CATIE, de $189 \mathrm{mg} / \mathrm{dl}$, foi superior ao valor de corte para SM (150 mg/dl); já a média de HDL sérico foi superior ao valor de corte para SM somente em mulheres (média de $47,7 \mathrm{mg} / \mathrm{dl}$ para mulheres e de $42,3 \mathrm{mg} / \mathrm{dl}$ para homens) (McEvoy et al., 2005). Cohn et al. (2004), contudo, observaram níveis mais elevados de triglicerídeos e níveis mais baixos de HDL em pacientes esquizofrênicos, quando comparados à população de referência. Dos pacientes avaliados em um estudo de prevalência de SM em pacientes com $\mathrm{TAB}, 48 \%$ apresentaram trigliceridemia $>150 \mathrm{mg} / \mathrm{dl}$ ou estavam em uso de drogas hipolipemiantes (Fagiolini $e t$ $a l .$, 2005). Todavia, nesse mesmo estudo, a porcentagem de homens com $\mathrm{HDL}<40 \mathrm{mg} / \mathrm{dl}$ ou de mulheres com $\mathrm{HDL}<50 \mathrm{mg} / \mathrm{dl}$ foi de apenas $23 \%$, valor inferior ao encontrado na população em geral.

\section{Discussão}

Os estudos expostos nesta revisão da literatura sugerem que pacientes portadores de transtornos mentais mais graves, como esquizofrenia e transtornos esquizoafetivos, apresentam probabilidade maior de serem acometidos por SM. O mesmo pode não ocorrer em se tratando de TAB, haja vista que, no único estudo envolvendo pacientes com esse transtorno, a prevalência de SM encontrada foi apenas discretamente superior à dos valores populacionais.

A maior prevalência de SM em populações psiquiátricas explica-se por uma causalidade multifatorial, em que se incluem fatores ligados ao estilo de vida desses pacientes, fatores genéticos, perinatais, neuroquímicos e hormonais, além do uso de psicofármacos. Sabe-se que alimentação inadequada e estilo de vida sedentário desempenham papéis importantes na gênese da SM (Zimmet et al., 2001). Indivíduos com esquizofrenia ou $\mathrm{TAB}$ realizam pouca atividade física e consomem dieta pouco saudável (Brown et al., 1999; Elmslie et al., 2001; Holt et al., 2004; Peet, 2004; Ryan e Thakore, 2002).

Variáveis psicossociais relacionadas ao estresse podem estar envolvidas. Sentimentos de cansaço excessivo ou traços de personalidade que refletem um estilo de vida estressante (comportamento tipo A, hostilidade $\mathrm{e}$ raiva) correlacionam-se significativamente com hiperinsulinemia, hiperglicemia, dislipidemia, hipertensão e obesidade central (Räikkönen et al., 1996). Um estudo de coorte de base populacional demonstrou que altos índices de sintomas depressivos, tensão e raiva em mulheres saudáveis predizem maior incidência de SM, após seguimento médio de 7,4 anos. Todavia, a interação no sentido inverso também foi constatada, já que a 
presença de SM, quando da avaliação inicial, também levou a aumento de sentimentos de raiva e ansiedade (Räikkönen et al., 2002).

Fatores genéticos e perinatais estão implicados nessa associação. Pacientes com esquizofrenia podem apresentar redução da atividade em lobo frontal de uma substância denominada brain-derived neurotrophic factor (BDNF), o que também está relacionado à menor sensibilidade à insulina (Peet, 2004). Estudos em populações orientais sugerem que polimorfismos de genes relacionados à insulina e à tirosina-hidroxilase associam-se tanto à resistência à insulina como à depressão (Ramasubbu, 2002). Contudo, a influência genética pode ser de menor importância em pacientes deprimidos. McCaffery et al. (2003) estudaram gêmeos monozigóticos e dizigóticos e concluíram que, na depressão, as influências ambientais respondem pela maior parte dessa associação. Por fim, o sofrimento perinatal é fator predisponente para desenvolvimento de resistência à insulina e também está implicado na gênese da esquizofrenia (Hofman et al., 2004; Holt et al., 2004).

Postula-se que o sistema serotoninérgico esteja envolvido na associação entre SM e depressão. A diminuição da função serotoninérgica associa-se com a ingestão aumentada de carboidratos, aos episódios bulímicos e à obesidade. Distúrbios na função serotoninérgica periférica, com aumento de receptores $5 \mathrm{HT}_{2} \mathrm{em}$ plaquetas, podem aumentar a agregação plaquetária e a vasoconstrição e contribuiriam para a trombogênese e a aterosclerose associadas à resistência à insulina (Holt et al., 2004).

A hipótese de hiperativação do eixo hipotálamo-pituitário-adrenal (HPA) tem sido proposta como um dos principais elos entre os transtornos mentais e a SM. A hipercortisolemia diminui a sensibilidade à insulina e se correlaciona com adiposidade central, hipertensão arterial, hipercolesterolemia e hipertrigliceridemia (Björntorp e Rosmond, 1999; Toalson et al., 2004). Estresse no início da vida ou história de estresse crônico, físico ou ambiental podem inibir os mecanismos de feedback que controlam a resposta cortisolêmica (Björntorp, 2001; Chrousos, 2000). Na depressão, a hiperatividade do eixo HPA talvez seja o achado bioquímico mais consistente (Ramasubbu, 2002). Já na esquizofrenia, diversas linhas de pesquisa também apontam para um eixo HPA hiper-reativo, com elevação da secreção de cortisol e um controle inibitório deficiente (Ryan e Thakore, 2002).

A avaliação de estudos que relacionam a incidência de SM ao uso de psicofármacos foge ao escopo desta revisão. Embora exista um vasto número de estudos sobre os efeitos adversos metabólicos dos psicofármacos, apenas recentemente começaram a surgir estudos que abordam especificamente o nexo causal entre psicofármacos e SM. Todavia, é razoável suspeitar que o uso de psicofármacos, principalmente antipsicóticos atípicos, seja provavelmente a causa mais importante da maior prevalência de $\mathrm{SM}$ em pacientes psiquiátricos. No estudo de Arranz et al. (2004), que compararam pacientes esquizofrênicos com ou sem história de uso de antipsicóticos, o uso prévio de antipsicóticos foi responsável pela maior resistência à insulina encontrada nesses pacientes. Recentemente, a Food and Drug Administration (FDA), nos Estados Unidos, determinou que a bula dos antipsicóticos atípicos contenha um alerta quanto ao risco de desenvolvimento de distúrbios do metabolismo da glicose (Citrome et al., 2005; Henderson, 2005). Todavia, cabe ressaltar que o risco para desenvolvimento de SM ou outros transtornos metabólicos não é o mesmo para todos os antipsicóticos (Citrome et al., 2005). Casey et al. (2004b) compararam pacientes em uso de aripiprazol $(n=504)$ e olanzapina $(n=505)$ para detectar a incidência ou a exacerbação de SM. Após 16 semanas, a ocorrência desses eventos foi de 8,5\% para aripiprazol versus $14,4 \%$ para olanzapina e, após um ano, de $10 \%$ para aripiprazol versus $20 \%$ para a olanzapina. O risco relativo para desenvolvimento de $\mathrm{SM}$ foi o dobro no grupo da olanzapina (RR: $2,1-\mathrm{p}=0,016$ ).

Ganho de peso induzido pelos psicofármacos é o principal fator que leva às disfunções metabólicas que ocorrem com o uso dessas drogas. Antidepressivos, principalmente os tricíclicos, lítio, ácido valpróico e diversos antipsicóticos estão associados a ganho significativo de peso (Casey, 2005; Garland et al., 1988). Entre os antipsicóticos atípicos, clozapina e olanzapina são as drogas que mais provocam ganho de peso (Allison et al., 1999b) e também as que mais se associam aos distúrbios do metabolismo da glicose e às dislipidemias (ADA et al., 2004). Embora a sedação secundária a algumas dessas drogas e a diminuição da atividade física estejam implicadas, os aumentos de apetite e ingestão calórica estão na base do surgimento desse efeito adverso (Casey, 2005; Elmslie et al., 2001; Garland et al., 1988; Holt et al., 2004; Sena et al., 2003; Zimmermann et al., 2003). Diversos psicofármacos agem sobre os centros nervosos ligados ao controle da saciedade e do peso, por meio do bloqueio de receptores histamínicos $\mathrm{H}_{1}$, receptores dopaminérgicos ou receptores serotoninérgicos 5- $\mathrm{HT}_{2 \mathrm{C}}$ (Henderson, 2005; Wirshing, 2004; Zimmermann et al., 2003). A hiperprolactinemia 
secundária a vários antipsicóticos também acarreta ganho de peso (Henderson, 2005).

Entrementes, é provável que alguns antipsicóticos interfiram diretamente na homeostase da glicose. Dados da FDA apontam que 25\% dos pacientes em uso dessas drogas que desenvolvem DM não apresentam obesidade nem ganho de peso significativo (Casey et al., 2004a). Ardizzone et al. (2001) demonstraram que a clozapina, a risperidona, a flufenazina, a loxapina e a amoxapina inibem o transporte de glicose em cultura de células PC-12 e em células musculares de ratos, de forma dose-dependente. $\mathrm{O}$ bloqueio de receptores $5 \mathrm{HT}_{1 \mathrm{~A}}$ das células beta das ilhotas pancreáticas e a inibição da liberação de insulina por receptores alfa ${ }_{2}$-adrenérgicos também têm sido apontados como possíveis causas nesse sentido (Citrome et al., 2005; Sena et al., 2003). Por fim, a hiperprolactinemia prolongada está associada a aumento do risco de DM (Henderson, 2005).

É importante notar que, apesar das altas taxas de SM em indivíduos esquizofrênicos, a prevalência aumentada de obesidade nessa população não foi constatada por Brown et al. (1999), na Inglaterra, ou por Allison et al. (1999a), nos Estados Unidos. Tais achados podem ser creditados à existência de porcentagem significativa de esquizofrênicos com baixo peso e à maior incidência de adiposidade visceral nessa população, independentemente da existência de obesidade (Ryan e Thakore, 2002). Theisen et al. (2001), pelo contrário, constataram prevalência elevada de obesidade em pacientes internados, o que pode ser consequiência do tratamento instituído, haja vista que taxas mais elevadas de obesidade foram observadas em pacientes em uso de antipsicóticos atípicos.

Os estudos encontrados que trataram da prevalência ou da incidência de SM e distúrbios metabólicos correlatos em pacientes deprimidos, com história de depressão ou escores elevados de sintomas depressivos, mostraram-se inconclusivos. Algumas hipóteses para explicar tais diferenças podem ser levantadas. A primeira hipótese diz respeito à perda de peso que, com freqüência, acompanha um episódio depressivo de maior gravidade e que pode levar à melhora de parâmetros metabólicos. A segunda hipótese, em sentido oposto, remete à parcela de indivíduos deprimidos que apresenta alterações no eixo HPA. Aproximadamente $40 \%$ dos pacientes com transtorno depressivo maior e $67 \%$ dos pacientes com depressão psicótica apresentam resposta cortisolêmica não supressora ao teste de supressão pela dexametasona (Brown et al., 2004). We-
ber-Hamann et al. (2002) demonstraram que pacientes deprimidos hipercortisolêmicos apresentam níveis glicêmicos mais elevados se comparados a deprimidos normocortisolêmicos. Thakore et al. (1997) constataram em mulheres deprimidas não obesas um aumento de adiposidade visceral, que se correlaciona também a níveis mais elevados de cortisol. No estudo prospectivo de Everson-Rose et al. (2004), a adiposidade visceral explicou a maior incidência de resistência à insulina $\mathrm{e}$ DM em pacientes deprimidas, o que vai ao encontro da possibilidade de haver um quadro subclínico de Cushing nessas pacientes.

Algumas limitações quanto aos resultados dessa revisão devem ser apontadas. Muitos dos estudos sobre prevalência de SM em populações psiquiátricas envolveram um pequeno número de pacientes. Ademais, em sua maioria, os valores encontrados foram obtidos de pacientes encaminhados a centros de referência ou a partir da avaliação inicial de pacientes incluídos em ensaios com psicofárma$\cos$. Assim, é possível que estes representem, na verdade, a prevalência de $\mathrm{SM}$ em pacientes com transtornos mentais de difícil tratamento. Há, contudo, exceções. O estudo de Kinder et al. (2004) pode ser considerado bastante representativo da população norte-americana, haja vista ter sido realizado sobre uma amostragem extensa, obtida a partir de dados do NHANES III; todavia, apresenta a limitação de ter sido realizado com indivíduos com história de depressão e de não poder ser generalizado para pacientes que estejam vivenciando um episódio depressivo. Por fim, a amostragem de indivíduos esquizofrênicos do estudo de Saari et al. (2005) foi realizada a partir de um banco de dados representativo da população local. Seus resultados, no entanto, devem ser encarados com reservas em razão do pequeno número de pacientes e da limitada faixa etária que representam.

\section{Conclusão}

Os estudos encontrados nesta revisão indicam prevalência aumentada de SM na população psiquiátrica, se comparada às taxas encontradas na população geral. Em se tratando de pacientes com esquizofrenia ou com transtorno esquizoafetivo, os estudos são concordantes em apontar para uma prevalência elevada de SM. Um único estudo envolvendo pacientes com $\mathrm{TAB}$ constatou prevalência discretamente elevada de SM.

É possível a existência de associação entre SM e história de depressão em mulheres. Um único estudo com amostragem extensa e representativa apresentou 
resultados que apontam nessa direção. Todavia, outras avaliações de prevalência ou incidência de distúrbios do metabolismo da glicose em pacientes deprimidos ou com escores elevados de sintomas depressivos apresentaram resultados contraditórios.

É de grande importância a realização de novas pesquisas sobre esse tema, com melhor delineação metodológica, utilizando amostras maiores e que incluam pacientes internados e ambulatoriais. Avaliações epidemiológicas com pacientes virgens de tratamento também devem ser realizadas, pois permitirão quantificar a influência do tratamento psicofarmacológico na etiologia da SM.

\section{Referências}

Alberti, K.G.M.M.; Zimmet, P.Z. for the WHO Consultation. - Definition, diagnosis and classification of diabetes mellitus and its complications, part 1: diagnosis and classification of diabetes melllitus. provisional report of a WHO consultation. Diabet Med 15: 539-553, 1998.

Allison, D.B.; Fontaine, K.R.; Heo, M.; Mentore, J.L.; Cappelleri, J.C.; Chandler $\mathrm{LP}$ et al. - The distribution of body mass index among individuals with and without schizophrenia. J Clin Psychiatry 60: 215-220, 1999a.

Allison, D.B.; Mentore, J.L.; Moonseong, H.; Chandler, L.; Cappelleri J.C.; Infante, M.C.; Weiden, P.J. - Antipsychotic-induced weight gain: a comprehensive research synthesis. Am J Psychiatry 156: 1686-2696, 1999b.

American Diabetes Association, American Psychiatric Association, American Association of Clinical Endocrinologists, North American Association for the Study of Obesity. - Consensus Development Conference on Antipsychotic Drugs and Obesity and Diabetes. J Clin Psychiatry 65: 267-272, 2004.

Ardizzone, T.D.; Bradley, R.J.; Freeman, A.M.; Dwyer, D.S. - Inhibition of glucose transport in $\mathrm{PC} 12$ cells by the atypical antipsychotic drugs risperidone and clozapine and structural analogs of clozapine. Brain Res 923: 82-90, 2001.

Arranz, B.; Rosel, P.; Ramírez, N.; Dueñas, R.; Fernández, P.; Sanchez, J.M.; Navarro, M.A.; San, L. - Insulin resistance and increased leptin concentrations in noncompliant schizophrenia patients but not in antipsychotic-naive first-episode schizophrenia patients. J Clin Psychiatry 65: 1335-1342, 2004.

Basu, R.; Brar, J.S.; Chengappa, K.N.R.; John, V.; Parepally, H.; Gershon, S. et al. - The prevalence of the metabolic syndrome in patients with schizoaffective disorder - bipolar subtype. Bipolar Disord 6: 314-318, 2004.

Bellnier, T.J. - The prevalence of metabolic disturbances in schizophrenic and bipolar I patients prior to antipsychotic use, $\ln : 156^{\text {th }}$ annual meeting of the American Psychiatric Association, 2003, San Francisco. Abstract 739.

Björntorp, P. - Do stress reactions cause abdominal obesity and comorbidities? Obesity Reviews 2: 73-86, 2001.

Björntorp, P.; Rosmond, R. - Hypothalamic origin of the metabolic syndrome X. Ann N Y Acad Sci 892: 297-307, 1999.

Brown, S. - Excess mortality of schizoprenia. Brit J Psychiatry 171: 502-508, 1997.

Brown, S.; Inskip, H.; Barraclough, B. - Causes of the excess mortality of schizophrenia. Br J Psychiatry 177: 212-217, 2000.

Brown, S.; Varghese, F.P.; McEwen, B.S. - Association of depression with medical illness: does cortisol play a role? Biol Psychiatry 55: 1-9, 2004.

Brown, S.; Birtwistle, J.; Roe, L.; Thompson, C. - The unhealthy lifestyle of people with schizophrenia. Psychol Med 29: 697-701, 1999.

Carnethon, M.R.; Kinder, L.S.; Fair, J.M.; Randall, S.S.; Fortmann, S.P. Symptons of depression as a risk factor for incident diabetes: findings from the National Health and Nutrition Examination Epidemiologic Follow-up Study, 1971-1992. Am J Epidemiol 158: 416-423, 2003.

Casey, D.E. - Metabolic issues and cardiovascular disease in patients with psychiatric disorders. Am J Med 118 suppl. 2: 15S-22S, 2005.

Casey, D.E.; Haupt, D.W.; Newcomer, J.W.; Henderson, D.C.; Sernyak, M.J.; Davidson, M. et al. - Antipsychotic-induced weight gain and metabolic abnormalities: implications for increased mortality in patients with schizophrenia. J Clin Psychiatry 65 suppl. 7: 4-16, 2004a.

Casey, D.E.; L'Italien, G.J.; Cislo, P. - Incidence of metabolic syndrome in olanzapine and aripiprazole patients. In: $157^{\text {th }}$ annual meeting of the American Psychiatric Association, 2004b; New York. Abstract 338.

Cassidy, F.; Ahearn, E.; Carroll, B.J. - Elevated frequency of diabetes mellitus in hospitalized manic-depressive patients. Am J Psychiatry 156: 1417-1420, 1999.

Chrousos, G.P. - The role of stress and the hypothalamic-pituitary-adrenal axis in the pathogenesis of the metabolic syndrome: neuro-endocrine and target tissue-related causes. Int J Obes Relat Metab Disord 24 suppl. 2: 50S-5S, 2000.

Citrome, L.; Blonde, L.; Damatarca, C. - Metabolic issues in patients with severe mental illness. South Med J 98: 714-720, 2005.

Cohen, D.; Stolk, R.P.; Grobbee, D.E.; Gispen de Wied, C.C. - Hyperglycemia and diabetes in patients with schizophrenia or schizoaffective disorders. Diabetes Care 29: 786-791, 2006.

Cohn, T.; Prud'homme, D.; Streiner, D.; Kameh, H.; Remington, G. - Characterizing coronary heart disease risk in chronic schizophrenia: high prevalence of metabolic syndrome. Can J Psychiatry 49: 753-760, 2004.

Correll, C.U.; Frederickson, A.M.; Kane, J.A.; Manu, P. - Metabolic syndrome and the risk of coronary heart disease in 367 patients treated with secondgeneration antipsychotic drugs. J Clin Psychiatry 67: 575-583, 2006.

Crabtree, L.H. - Offsetting metabolic abnormalities and premature death in medicated patients. In: $157^{\text {th }}$ annual meeting of the American Psychiatry Association, 2004; New York. Abstract 545.

Davidson, K.; Jonas, B.S.; Kim, E.; Dixon, R.N.; Markovitz, J.H. - Do depression symptons predict early hypertension incidence in young adults in the CARDIA study? Arch Inter Med 160: 1495-500, 2000.

De Hert, M.A.; van Winkel, R.; Van Eyck, D.; Hanssens, L.; Wampers, M.; Scheen, A.; Peuskens, J. - Prevalence of the metabolic syndrome in patients with schizophrenia treated with antipsychotic medication. Schizophr Res 83: 87-93, 2006.

Dixon, L.; Weiden, P.; Delahanty, J.; Goldberg, R.; Postrado, L.; Lucksted, A.; Lehman, A. - Prevalence and correlates of diabetes in national schizophrenia samples. Schizophr Bull 26: 903-912, 2000.

Eaton, W.W.; Armenian, H.; Gallo, J.; Pratt, L.; Ford, D.E. - Depression and risk for onset of type II diabetes: a prospective population-based study. Diabetes Care 19: 1097-1102, 1996.

Elmslie, J.L.; Mann, J.I.; Silverstone, J.T.; Williams, S.M.; Romans, S.E. - Determinants of overweight and obesity in patients with bipolar disorder. J Clin Psychiatry 62: 486-491, 2001.

Elmslie, J.; Silverstone, J.T.; Mann, J.I.; Williams, S.M.; Romans, S.E. - Prevalence of overweight and obesity in bipolar patients. J Clin Psychiatry 61: 179-184, 2000.

Everson-Rose, S.A.; Meyer, P.M.; Powell, L.H.; Pandey, D.; Torréns, J.I.; Kravitz, H.M. et al. - Depressive symptons, insulin resistance, and risk of diabetes in women at midlife. Diabetes Care 27: 2856-2862, 2004.

Executive summary of the Third Report of the National Cholesterol Education Program (NCEP) Expert Panel on Detection, Evaluation and Treatment of High Blood Cholesterol in Adults (Adult Treatment Panel III). JAMA 285: 2486-2497, 2001.

Fagiolini, A.; Frank, E.; Houck, P.R.; Mallinger, A.G.; Swartz, H.A.; Buysse, D.J. et al. - Prevalence of obesity and weight change during treatment in patients with bipolar I disorder. J Clin Psychiatry 63: 528-533, 2000.

Fagiolini, A.; Frank, E.; Scott, J.A.; Turkin, S.; Kupfer, D.J. - Metabolic syndrome in bipolar disorder: findings from the Bipolar Disorder Center for Pennsylvanians. Bipolar Disord 7: 424-430, 2005.

Fava, M. - Weight gain and antidepressants. J Clin Psychiatry 61 suppl. 11: 37-41, 2000.

Ford, E.S.; Giles, W.H.; Dietz, W.H. - Prevalence of the metabolic syndrome among US adults. Findings from the third national health and nutrition examination survey. Jama 287: 356-359, 2002.

Garland, E.J.; Remick, R.A.; Zis, A.P. - Weight gain with antidepressants and lithium. J Clin Psychopharmacol 8: 323-330, 1988.

Grundy, S.M.; Brewer, B.; Cleeman, J.I.; Smith, S.C.; Lenfant, C. - Definition of metabolic syndrome. Report of the National Heart, Lung, and Blood Instittute/American Heart Association Conference on Scientific Issues Related to Definition. Circulation 109: 133-138, 2004. 
Hagg, S.; Lindblom, Y.; Mjorndal, T.; Adolfsson, R. - High prevalence of the metabolic syndrome among a Swedish cohort of patients with schizophrenia. Int Clin Psychopharmacol 21: 93-98, 2006.

Harris, E.C.; Barraclough, B. - Excess of mortality of mental disorder. Brit J Psychiatry 173: 11-52, 1988.

Heiskanen, T.; Niskanen, L.; Lyytikäinen, R.; Saarinen, P.I.; Hintikka, J. - Metabolic syndrome in patients with schizophrenia. J Clin Psychiatry 64: 575-579, 2003.

Henderson, D.C. - Schizophrenia and comorbid metabolic disorders. J Clin Psychiatry 66 suppl. 6: 11-20, 2005.

Hofman, P.L.; Regan, F.; Jackson, W.E.; Jefferies, C.; Knight, D.B.; Robinson, E.M.; Cutfield, W.S. - Premature birth and later insulin resistance. $N$ Engl J Med 351: 2179-2186, 2004.

Holt, R.I.G.; Peveler, R.C.; Byrne, C.D. - Schizophrenia, the metabolic syndrome and diabetes. Diabet Med 21: 515-523, 2004.

International Diabetes Federation. The IDFworldwide definition of the metabolic syndrome. Disponível em: www.idf.org/home/index.cfm?unode=1120071EAACE-41D2-9FA0-BAB6E25BA072. Acesso em 03/07/2005.

Jeste, D.V.; Gladsjo, J.A.; Lindamer, L.A.; Lacro, J.P. - Medical comorbidity in schizophrenia. Schizophr Bull 22: 413-430, 1996.

Jonas, B.S.; Frank, P.; Ingram, D.D. - Are symptoms of anxiety and depression risk factors for hypertension? Longitudinal evidence from the National Health and Nutrition Examination Survey I Epidemiology Follow-up Study. Arch Fam Med 6: 43-49, 1997. Abstract.

Kato, M.M.; Currier, M.B.; Gomez, C.M.; Hall, L.; Gonzalez-Blanco, M. - Prevalence of metabolic syndrome in hispanic and non-hispanic patients with schizophrenia. Prim Care Companion J Clin Psychiatry 6: 74-77, 2004.

Kinder, L.S.; Carnethon, M.R.; Palaniappan, L.P.; King, A.C.; Fortmann, S.P. - Depression and the metabolic syndrome in young adults: findings from the Third National Health and Nutrition Examination Survey. Psychosom Med 66: 316-322, 2004.

Lawlor, D.A.; Smith, G.D.; Ebrahim, S. - Association of insulin resistance with depression: cross sectional findings from the British women's heart and health study. BMJ 327: 1383-1384, 2003.

Lawlor, D.A.; Ben-Shlomo, Y.; Ebrahim, S.; Smith, G.D.; Stansfeld, S.A.; Yarnell, J.W.G.; Gallacher, J.E.J. - Insulin resistance and depressive symptons in middle aged men: findings from the Caerphilly prospective cohort study. BMJ 330: 705-706, 2005.

Lett, H.S.; Blumenthal, J.A.; Babyak, M.A.; Sherwood, A.; Strauman, T.; Robins, C.; Newman, M.F.-Depression as a risk factor for coronary artery disease: evidence, mechanisms, and treatment. Psychosom Med 66: 305-315, 2004.

Littrell, K.H.; Petty, R.G.; Ortega, T.R.; Moore, D.; Ballard, A.; Clough, R. et al. - Insulin resistance and syndrome $X$ among patients with schizophrenia. In: $156^{\text {th }}$ annual meeting of the American Psychiatric Association, 2003; San Francisco. Abstract 550.

McCaffery, J.M.; Niaura, R.; Todard, J.F.; Swan, G.E.; Carmelli, D. - Depressive symptons amd metabolic risk in adult male twins enrolled in the National Heart, Lung, and Blood Institute Twin Study. Psychosom Med 65: 490-497, 2003.

McElroy, S.L.; Kotwal, R.; Malhotra, S.; Nelson, E.B.; Keck, P.E.; Nemeroff, C.B. - Are mood disorders and obesity related? A review for the mental health professional. J Clin Psychiatry 65: 634-651, 2004.

McEvoy, J.P.; Meyer, J.M.; Goff, D.C.; Nasrallah, H.A.; Davis, S.M.; Sullivan, L. et al. - Prevalence of the metabolic syndrome in patients with schizophrenia: baseline results from the Clinical Antipsychotic Trials of Intervention Effectiveness (CATIE) schizophrenia trial and comparison with national estimates from NHANES III. Schizophr Res 80: 19-32, 2005.

Meyer, J.; Loh, C.; Leckband, S.G.; Boyd, J.A.; Wirshing, W.C.; Pierre, J.M.; Wirshing, D. - Prevalence of the metabolic syndrome in veterans with schizophrenia. J Psychiatr Pract 12: 5-10, 2006.

Mukherjee, S.; Decina, P.; Bocola, V.; Saraceni, F; Scapicchio, P.L. - Diabetes mellitus in schizophrenic patients. Compr Psychiatry 37: 68-73, 1996.

Murphy, J.M.; Monson, R.R.; Olivier. D.C.; Sobol, A.M.; Leighton, A.H. - Affective disorders and mortality: a general population study. Arch Gen Psychiatry 44: 473-480, 1987.

Okamura, F.; Tashiro, A.; Utumi, A.; Towako, I.; Takatosi, S.; Tamura, D. et al. - Insulin resistance in patients with depression and its changes during the clinical course of depression: minimal model analysis. Metabolism 49: 1255-1260, 2000.
Pandina, G.J.; Greenspan, A.; Bossie, C.; Turkoz, I.; Morein, J.; Meyer, J.M. - Metabolic syndrome in patients with schizophrenia. In: $157^{\text {th }}$ annual meeting of the American Psychiatric Association, 2004; New York. Abstract 584.

Peet, M. - Diet, diabetes and schizophrenia: review and hypothesis. $\mathrm{Br} \mathrm{J}$ Psychiatry 184 suppl. 47: 102S-5S, 2004.

Räikkönen, K.; Mattheus, K.A.; Kuller, L.H. - The relationship between psychological risk attributes and the metabolic syndrome in healthy women: antecedent or consequence? Metabolism 51: 1573-1577, 2002.

Räikkönen, K.; Keltikangas-Järvinen, L.; Adlercreutz, H.; Hautanen, A. -Psychosocial stress and the insulin resistance syndrome. Metabolism 45: 1533-1538, 1996.

Ramasubbu, R. - Insuline resistance: a metabolic link between depressive disorder and atherosclerotic vascular diseases. Medical Hypothesis 59: 537-551, 2002.

Reaven, G.M. - Syndrome X: 6 years later. J Int Med 236 suppl. 736: 13-22, 1994.

Regenold, W.T.; Thapar, R.K.; Marano, C.; Gavirneni, S.; Kondapavuluru, P.V. - Increased prevalence of type 2 diabetes mellitus among psychiatric inpatients with bipolar I affective and schizoaffective disorders independent of psychotropic drug use. J Affect Disord 70: 19-26, 2002.

Rosenbaum, P.; Ferreira, S.R.G. - An update on cardivascular risk of metabolic syndrome. Arq Bras Endocrinol Metab 47: 220-227, 2003.

Ryan, M.C.M.; Thakore, J.H. - Physical consequences of schizophrenia and its treatment - the metabolic syndrome. Life Sciences 71: 239-257, 2002.

Ryan, M.C.M.; Collins, P.; Thakore, J.H. - Impaired fasting glucose tolerance in first-episode drug-naive patients with schizophrenia. Am J Psychiatry 160: 284-289, 2003.

Saari, K.M.; Sari, M.L.; Villo, K.M.; Isohanni, M.K.; Järvelin, M.R.; Laurén, L.H. et al. - A 4-fold risk of metabolic syndrome in patients with schizophrenia: the Northern Finland 1966 Birth Cohort Study. J Clin Psychiatry 66: 559-563, 2005.

Sena, E.P.; Sampaio, A.S.; Quarantini, L.C.; Oliveira, I.R. - Diabetes mellitus e antipsicóticos atípicos. Rev Bras Psiquiatr 25: 253-257, 2003.

Sociedade Brasileira de Hipertensão, Sociedade Brasileira de Cardiologia, Sociedade Brasileira de Endocrinologia e Metabologia, Sociedade Brasileira de Diabetes, Associação Brasileira para Estudos da Obesidade. I Diretriz Brasileira de Diagnóstico e Tratamento da Síndrome Metabólica. Hipertensão 7: 124-159, 2004.

Straker, D.; Correll, C.U.; Kramer-Ginsberg, E.; Abdulhamid, N.; Koshy, F.; Rubens, E. et al. - Cost-effective screening for the metabolic syndrome in patients treated with second-generation antipsychotic medications. Am J Psychiatry 162: 1217-1221, 2005.

Subramaniam, M.; Chong, S.A.; Pek, E. - Diabetes mellitus and impaired glucose tolerance in patients with schizophrenia. Can J Psychiatry 48: 345-347, 2003.

Susce, M.T.; Vilanueva, N.; Diaz, F.J.; Leon, J. - Obesity and associated complications in patients with severe mental illness: a cross-sectional survey. J Clin Psychiatry 66: 167-173, 2005.

Thakore, J.H.; Richards, P.J.; Reznek, R.H.; Martin, A.; Dinan, T.G. - Increased intra-abdominal fat deposition in patients with major depressive illness as measured by computed tomography. Biol Psychiatry 41: 1140-1142, 1997.

Timonen, M.; Laakso, M.; Jokelainen, J.; Rajala, U.; Meyer-Rochow, V.B.; Keinänen-Kiukaanniemi, S. - Insulin resistance and depression: cross sectional study. BMJ 330: 17-18, 2005.

Theisen, F.M.; Linden, A.; Geller, F.; Schafer, H.; Martin, M.; Remschmidt, H.; Hebebrand, J. - Prevalence of obesity in adolescent and young adult patients with and without schizophrenia and in relationship to antipsychotic medication. J Psychiatr Res 35: 339-345, 2001.

Toalson, P.; Ahmed, S.; Hardy, T.; Kabinoff, G. - The metabolic syndrome in patients with severe mental illnesses. Prim Care Companion 6: 152-158, 2004.

Weber-Hamann, B.; Hentschel, F.; Kniest, A.; Deuschle, M.; Colla, M.; Lederbogen, F.; Heuser, I. - Hypercotisolemic depression is associated with increased intra-abdominal fat. Psychosom Med 64: 274-277, 2002.

Wirshing, D. - Schizophrenia and obesity: impact of antipsychotic medications. J Clin Psychiatry 65 suppl. 18: 13-26, 2004.

Zimmermann, U.; Thomas, K.; Himmerich, H.; Schuld, A.; Pollmächer, T. -Epidemiology, implications and mechanisms underlying drug-induced weight gain in psychiatric patients. J Psychiatr Res 37: 193-220, 2003.

Zimmet, P.; Albertl, K.G.M.M.; Shaw, J. - Global and societal implications of diabetes epidemic. Nature 414: 782-787, 2001. 